\title{
Mapping the Landscape of Human-Level Artificial General Intelligence
}

\author{
Sam S. Adams, Itamar Arel, Joscha Bach, Robert Coop, Rod Furlan, \\ Ben Goertzel, J. Storrs Hall, Alexei Samsonovich, Matthias Scheutz, \\ Matthew Schlesinger, Stuart C. Shapiro, John F. Sowa
}

We present the broad outlines of a roadmap toward human-level artificial general intelligence (henceforth, AGI). We begin by discussing AGI in general, adopting a pragmatic goal for its attainment and a necessary foundation of characteristics and requirements. An initial capability landscape will be presented, drawing on major themes from developmental psychology and illuminated by mathematical, physiological, and information-processing perspectives. The challenge of identifying appropriate tasks and environments for measuring AGI will be addressed, and seven scenarios will be presented as milestones suggesting a roadmap across the AGI landscape along with directions for future research and collaboration.
$\mathrm{T}$ his article is the result of an ongoing collaborative effort by the coauthors, preceding and during the AGI Roadmap Workshop held at the University of Tennessee, Knoxville in October 2009, and from many continuing discussions since then. Some of the ideas also trace back to discussions held during two Evaluation and Metrics for Human Level AI workshopa organized by John Laird and Pat Langley (one in Ann Arbor in late 2008, and one in Tempe in early 2009). Some of the conclusions of the Ann Arbor workshop were recorded (Laird et al. 2009). Inspiration was also obtained from discussion at the Future of AGI postconference workshop of the AGI-09 conference, triggered by Itamar Arel's presentation AGI Roadmap (Arel 2009); and from an earlier article on AGI road-mapping (Arel and Livingston 2009).

Of course, this is far from the first attempt to plot a course toward human-level AGI: arguably this was the goal of the founders of the field of artificial intelligence in the 1950s, and has been pursued by a steady stream of AI researchers since, even as the majority of the AI field has focused its attention on more narrow, specific subgoals. The ideas presented here build on the ideas of others in innumerable ways, but to review the history of AI and situate the current effort in the context of its predecessors would require a much longer article than this one. Thus we have chosen to focus on the results of our AGI roadmap discussions, acknowledging in a broad way the many debts owed to many prior researchers. References to the prior literature on evaluation of advanced AI systems are given by Laird (Laird et al. 2009) and Geortzel and Bugaj (2009), which may in a limited sense be considered prequels to this article.

We begin by discussing AGI in general and adopt a pragmatic goal for measuring progress toward its attainment. We also 
adopt, as a provisional starting point, a slightly modified version of the characteristics and requirements for AGI proposed by Laird and Wray (2010), upon which we will later construct a number of specific scenarios for assessing progress in achieving AGI. An initial capability landscape for AGI will be presented, drawing on major themes from developmental psychology and illuminated by mathematical, physiological, and informationprocessing perspectives. The challenge of identifying appropriate tasks and environments for measuring AGI will be taken up. Several scenarios will be presented as milestones outlining a roadmap across the AGI landscape, and directions for future work and collaboration will conclude the article.

\section{The Goal: Human-Level General Intelligence}

Simply stated, the goal of AGI research as considered here is the development and demonstration of systems that exhibit the broad range of general intelligence found in humans. This goal of developing AGI echoes that of the early years of the artificial intelligence movement, which after many valiant efforts largely settled for research into narrow AI systems that could demonstrate or surpass human performance in a specific task, but could not generalize this capability to other types of tasks or other domains.

A classic example of the narrow AI approach was IBM's Deep Blue system (Campbell, Hoane, and Hsu 2002), which successfully defeated world chess champion Gary Kasparov but could not readily apply that skill to any other problem domain without substantial human reprogramming. In early 2011, IBM's Watson question-answering system (Ferrucci 2010) dramatically defeated two all-time champions in the quiz show Jeopardy, but having never personally visited Chicago's O'Hare and Midway airports, fumbled on a question that any human frequent flier in the US would have known. To apply the technology underlying Watson to another domain, such as insurance or call-center support, would require not merely education of the AI system, but significant reprogramming and human scoring of relevant data - the analogue of needing to perform brain surgery on a human each time the person needs to confront a new sort of task. As impressive as these and other AI systems are in their restricted roles, they all lack the basic cognitive capabilities and common sense of a typical five-year-old child, let alone a fully educated adult professional.

Given the immense scope of the task of creating AGI, we believe the best path to long-term success is collaboration and coordination of the efforts of multiple research groups. A common goal and a shared understanding of the landscape ahead of us will be crucial to that success, and it was the aim of our workshop to make substantial progress in that direction.

\section{A Pragmatic Goal for AGI}

The heterogeneity of general intelligence in humans makes it practically impossible to develop a comprehensive, fine-grained measurement system for AGI. While we encourage research in defining such high-fidelity metrics for specific capabilities, we feel that at this stage of AGI development a pragmatic, high-level goal is the best we can agree upon.

Nils Nilsson, one of the early leaders of the AI field, stated such a goal in the 2005 AI Magazine article Human-Level Artificial Intelligence? Be Serious! (Nilsson, 2005):

I claim achieving real human-level artificial intelligence would necessarily imply that most of the tasks that humans perform for pay could be automated. Rather than work toward this goal of automation by building special-purpose systems, I argue for the development of general-purpose, educable systems that can learn and be taught to perform any of the thousands of jobs that humans can perform. Joining others who have made similar proposals, I advocate beginning with a system that has minimal, although extensive, built-in capabilities. These would have to include the ability to improve through learning along with many other abilities.

Many variant approaches have been proposed for achieving such a goal, and both the AI and AGI communities have been working for decades on the myriad subgoals that would have to be achieved and integrated to deliver a comprehensive AGI system. But aside from the many technological and theoretical challenges involved in this effort, we feel the greatest impediment to progress is the absence of a common framework for collaboration and comparison of results. The AGI community has been working on defining such a framework for several years now, and as mentioned earlier, this present effort on AGI road-mapping builds on the shoulders of much prior work.

\section{Characteristics and Requirements for AGI}

As a starting point for our road-mapping effort, we have adopted a slightly modified version of Laird and Wray's Cognitive Architecture Requirements for Achieving AGI (Laird and Wray 2010). Their outline of eight characteristics for environments, tasks, and agents and 12 requirements for general cognitive architectures provides a level foundation for the comparison of appropriate scenarios for assessing AGI systems. We stress, however, that in our perspective these requirements should not be considered as final or set in stone, but simply as a convenient point of departure for discussion and collaboration. As our understanding of AGI improves through ongoing research, our under- 
standing of the associated requirements is sure to evolve. In all probability, each of the many current AGI research paradigms could be used to spawn a slightly different requirements list, but we must start somewhere if we are to make progress as a community.

To test the capability of any AGI system, the characteristics of the intelligent agent and its assigned tasks within the context of a given environment must be well specified. Failure to do this may result in a convincing demonstration, but make it exceedingly difficult for other researchers to duplicate experiments and compare and contrast alternative approaches and implementations.

The characteristics shown in figure 1 provide the necessary (if not sufficient) degrees of dynamism and complexity that will weed out most narrow AI approaches at the outset, while continually challenging researchers to consider the larger goal of AGI during their work on subsystems and distinct capabilities. We have added the requirement for openness of the environment (C2), that is, the agent should not be able to rely on a fixed library of objects, relations, and events. We also require objects to have an internal structure that requires complex, flexible representations (C1).

There are nearly as many different AGI architectures as there are researchers in the field. If we are ever to be able to compare and contrast systems, let alone integrate them, a common set of architectural features must form the basis for that comparison.

Figure 2 reprises Laird and Wray's requirements for general cognitive architectures and provides a framework for that basis. We have modified requirement $\mathrm{R} 0$, which in Laird and Wray reads "fixed structure for all tasks," to emphasize that the system may grow and develop over time, perhaps changing its structure dramatically - but these changes need to be effected by the agent itself, not by the intervention of the programmer. We anticipate further additions and changes to these lists over time as the community converges in experience with various cognitive architectures and AGI systems, but we have adopted them so we can progress as a community to our larger, shared goals.

\section{Current Challenges}

In addition to providing a framework for collaborative research in AGI, Laird and Wray (2010) identified two significant challenges:

... one of the best ways to refine and extend these sets of requirements and characteristics is to develop agents using cognitive architectures that test the sufficiency and necessity of all these and other possible characteristics and requirements on a variety of real-world tasks. One challenge is to find tasks and environments where all of these characteristics are active, and thus all of the requirements must be
C1. The environment is complex, with diverse, interacting and richly structured objects.

C2. The environment is dynamic and open.

C3. Task-relevant regularities exist at multiple time scales.

C4. Other agents impact performance.

C5. Tasks can be complex, diverse and novel.

C6. Interactions between agent, environment and tasks are complex and limited.

C7. Computational resources of the agent are limited.

C8. Agent existence is long-term and continual.

Figure 1. Characteristics for AGI Environments, Tasks, and Agents.

R0. New tasks do not require re-programming of the agent

R1. Realize a symbol system

Represent and effectively use:

R2. Modality-specific knowledge

R3. Large bodies of diverse knowledge

R4. Knowledge with different levels of generality

R5. Diverse levels of knowledge

R6. Beliefs independent of current perception

R7. Rich, hierarchical control knowledge

R8. Meta-cognitive knowledge

R9. Support a spectrum of bounded and unbounded deliberation

R10. Support diverse, comprehensive learning

R11 Support incremental, online learning

Figure 2. Cognitive Architecture Requirements for AGI.

confronted. A second challenge is that the existence of an architecture that achieves a subset of these requirements does not guarantee that such an architecture can be extended to achieve other requirements while maintaining satisfaction of the original set of requirements.

Our 2009 AGI road-mapping workshop took up the first challenge of finding appropriate tasks and 
environments to assess AGI systems, while the second challenge will be more appropriately handled by individual research efforts. We also added a third challenge, that of defining the landscape of AGI, in service to the AGI road-mapping effort that has been under way for several years.

The balance of this article will deal with these two challenges in reverse order. First, we will provide an initial landscape of AGI capabilities based on developmental psychology and underpinned by mathematical, physiological, and informationprocessing perspectives. Second, we will discuss the issues that arise in selecting and defining appropriate tasks and environments for assessing AGI, and finally, we will present a number of scenarios and directions for future work and collaboration.

\section{Challenge: Mapping the Landscape}

There was much discussion both in preparation and throughout our AGI Roadmap Workshop on the process of developing a roadmap. A traditional highway roadmap shows multiple driving routes across a landscape of cities and towns, natural features like rivers and mountains, and political features like state and national borders. A technology roadmap typically shows a single progression of developmental milestones from a known starting point to a desired result. Our first challenge in defining a roadmap for achieving AGI was that we initially had neither a well-defined starting point nor a commonly agreed upon target result. The history of both AI and AGI is replete with this problem, which is somewhat understandable given the breadth and depth of the subjects of both human intelligence and computer technology. We made progress by borrowing more metaphors from the highway roadmap, deciding to first define the landscape for AGI and then populate that landscape with milestones that may be traversed through multiple alternative routes.

The final destination, full human-level artificial general intelligence, encompasses a system that could learn, replicate, and possibly exceed humanlevel performance in the full breadth of cognitive and intellectual abilities. The starting point, however, was more problematic, since there are many current approaches to achieving AGI that assume different initial states. We finally settled on a developmental approach to the roadmap, following human cognitive development from birth through adulthood.

The various scenarios for assessing AGI that had been submitted by participants prior to the workshop were then arrayed as milestones between these two endpoints, without any specific routes between them aside from their relative requirements for increasing levels of human cognitive development.

\section{Top Down: Characterizing Human Cognitive Development}

The psychological approach to intelligence encompasses a broad variety of subapproaches rather than presenting a unified perspective. Viewed historically, efforts to conceptualize, define, and measure intelligence in humans reflect a distinct trend from general to specific (Gregory 1996), much like the history of AI.

Early work in defining and measuring intelligence was heavily influenced by Spearman, who in 1904 proposed the psychological factor g (for general intelligence). Spearman argued that $g$ was biologically determined, and represented the overall intellectual skill level of an individual. A related advance was made in 1905 by Binet and Simon, who developed a novel approach for measuring general intelligence in French schoolchildren. A unique feature of the Binet-Simon scale was that it provided comprehensive age norms, so that each child could be systematically compared with others across both age and intellectual skill level. In 1916, Terman introduced the notion of an intelligence quotient or IQ, which is computed by dividing the test taker's mental age (that is, his or her age-equivalent performance level) by the physical or chronological age.

In subsequent years, psychologists began to question the concept of intelligence as a single, undifferentiated capacity. There were two primary concerns. First, while performance within an individual across knowledge domains is somewhat correlated, it is not unusual for skill levels in one domain to be considerably higher or lower than in another (that is, intraindividual variability). Second, two individuals with comparable overall performance levels might differ significantly across specific knowledge domains (that is, interindividual variability).

These issues helped to motivate a number of alternative theories, definitions, and measurement approaches, which share the idea that intelligence is multifaceted and variable both within and across individuals. Of these approaches, a particularly well-known example is Gardner's theory of multiple intelligences (Gardner 1999), which proposes eight distinct forms or types of intelligence: (1) linguistic, (2) logical-mathematical, (3) musical, (4) bodily-kinesthetic, (5) spatial, (6) interpersonal, (7) intrapersonal, and (8) naturalist. Gardner's theory suggests that each individual's intellectual skill is represented by an intelligence profile, a unique mosaic or combination of skill levels across the eight forms of intelligence.

While Gardner's theory has had significant impact within the field of adult intelligence, it has had comparatively less influence on the study of intellectual development in children. Instead, researchers in the field of cognitive development 
seek to (1) describe processes of intellectual change, while (2) identifying and explaining the underlying mechanisms (both biological and environmental) that make these changes possible. Contemporary theories of cognitive development are very diverse and defy simple systematization, and a thorough treatment of the field would take us too far from our focus. However, two major schools of thought, those of Piaget and Vygotsky, will serve as axes for our AGI landscape.

\section{Piaget's Theory}

In his classic work that founded the science of cognitive development, Piaget proposed that humans progress through four qualitatively distinct stages (Piaget 1953).

First, during the sensorimotor stage (0-2 years), infants acquire a rich repertoire of perceptual and motor skills (for example, reaching, grasping, crawling, walking, and others). This stage includes a number of major milestones, such as the ability to search for hidden objects and the ability to use objects as simple tools.

Second, infants enter the preoperational stage (2-6 years) as they acquire the capacity to mentally represent their experiences (for example, memory, mental images, drawing, language, and others), but lack the ability to systematically coordinate these representations in a logically consistent manner.

During the next stage (6 years to adolescence), children at the concrete operational level master basic elements of logical and mathematical thought, including the ability to reason about classes and categories, as well as numerical operations and relations.

The final stage of development, formal operations, begins in adolescence and includes the use of deductive logic, combinatorial reasoning, and the ability to reason about hypothetical events.

\section{Vygotsky's Theory}

In contrast to Piaget's view, which focuses on the individual, Vygotsky's classic theory of cognitive development emphasizes the sociocultural perspective (Vygotsky 1986). Vygotsky's theory not only highlights the influence of the social environment, but it also proposes that each culture provides a unique developmental context for the child. Three fundamental concepts from this theory are (1) internalization, (2) zone of proximal development, and (3) tools of intellectual adaptation.

First, Vygotsky proposed that the capacity for thought begins by acquiring speech (that is, thinking out loud), which gradually becomes covert or internalized.

Second, he emphasized that parents, teachers, and skilled peers facilitate development by helping the child function at a level just beyond what he or she is capable of doing alone; he referred to this cognitive space as the zone of proximal development.

Third, Vygotsky also stressed that each child inherits a unique set of objects, ideas, and traditions that guide learning (for example, books, calculators, computers, and others). These tools of intellectual adaptation not only influence the pattern of cognitive development, but also serve as constraints on the rate and extent of development.

\section{Surveying the Landscape} of Human Intelligence

While many consider the views of Piaget and Vygotsky to be at odds because of their different foci, we consider them to be complementary, partial descriptions of the same development process. Piaget's approach focused on the stages of cognitive development of an individual child, while Vygotsky considered the same development within the context of social interaction with other humans with access to and training in the use of cultural artifacts like tools, languages, books, and a shared environment. By placing the developmental stages of each theory on opposing axes (figure 3 ), we can outline the landscape of human cognitive development and provide a structure for the placement of milestones along the road to AGI.

\section{Bottom Up: Substrata of the AGI Landscape}

Our ultimate goal as a community is to create functional computer systems that demonstrate humanlevel general intelligence. This lofty aim requires that we deeply understand the characteristics of human cognitive behavior and development outlined above, as well as implement that understanding in a nonbiological substrate, the modern digital computer. To accomplish this, we draw inspiration and perspectives from many different disciplines, as shown in figure 4 . From physiology we seek to understand the biological implementation of human intelligence; from mathematics, the nature of information and intelligence regardless of its implementation; and from information processing, we map these insights and others into the metaphors of computer science that will most directly lead to successful computer implementation. This section describes each of these underpinnings for our road-mapping efforts.

\section{The Physiological Perspective}

Given advances in bioscience, it has become increasingly feasible to take a more holistic physiological perspective on cognitive development incorporating genetic, biochemical, and neural mechanisms, among others. A unique strength of biologically inspired cognitive theories is their ability to account for universal aspects of development 


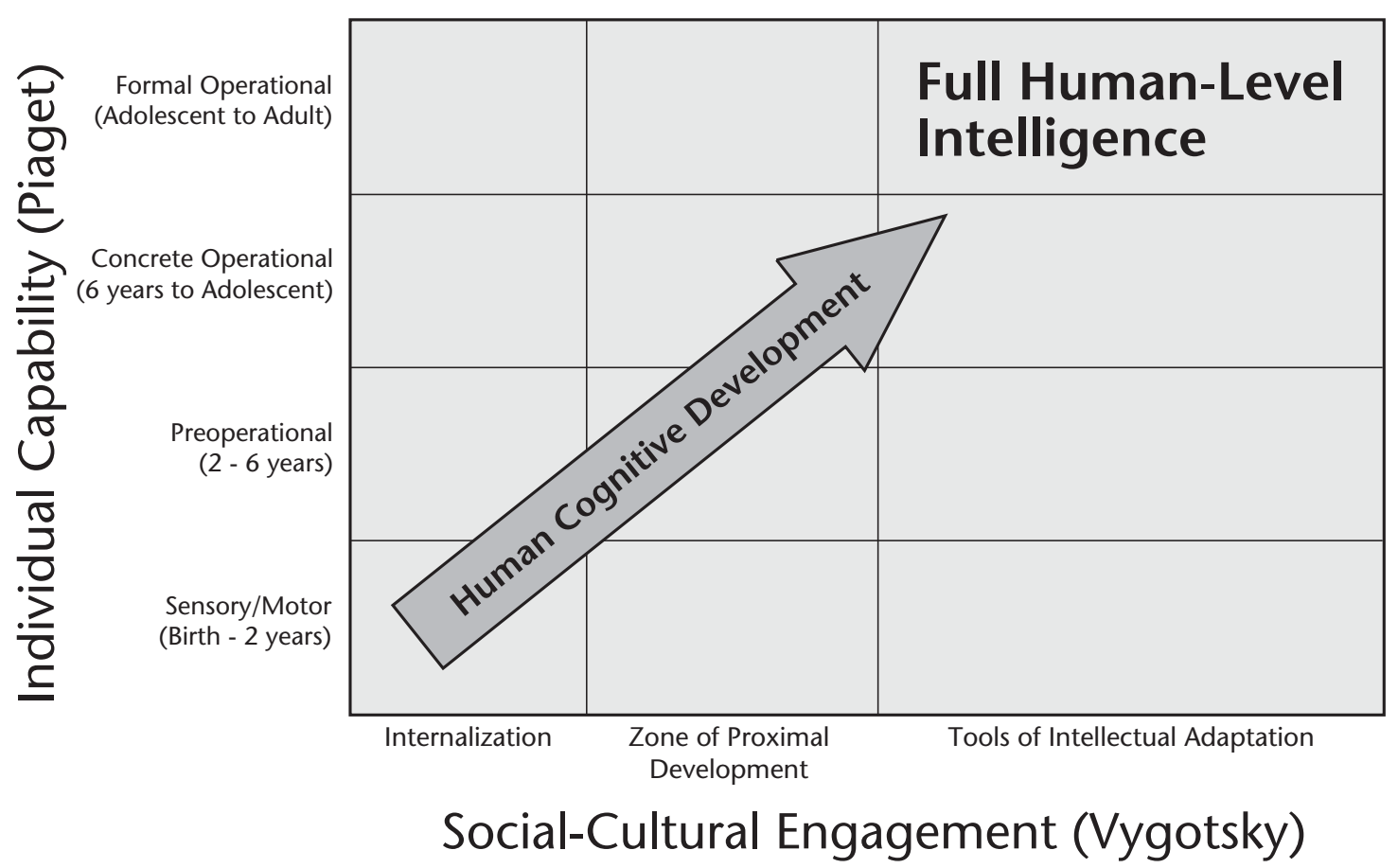

Figure 3. The Landscape of Human Cognitive Development.

that emerge in a consistent pattern across a wide range of cultures, physical environments, and historical time periods (for example, motor-skill development, object perception, language acquisition, and others). The physiological approach also plays a prominent role in explaining differences between typical and atypical patterns of development (for example, autism, ADHD, learning disorders, disabilities, and others). Both physiological and information-processing perspectives favor modular accounts of cognitive development, which view the brain as divided into special-purpose input-output systems that are devoted to specific processing tasks.

\section{The Mathematical Perspective}

Thie mathematical perspective is typified by the recent work of Marcus Hutter and Shane Legg, who give a formal definition of general intelligence based on the Solomonoff-Levin distribution (Legg and Hutter 2007). Put very roughly, they define intelligence as the average reward-achieving capability of a system, calculated by averaging over all possible reward-summable environments, where each environment is weighted in such a way that more compactly describable programs have larger weights. Variants of this definition have been pro- posed to take into account the way that intelligence may be biased to particular sorts of environments, and the fact that not all intelligent systems are explicitly reward-seeking (Goertzel 2010).

While this notion of intelligence as compression is useful, even if a mathematical definition of general intelligence were fully agreed upon, this wouldn't address the human-level part of AGI. Human intelligence is neither completely general in the sense of a theoretical AGI like AIXI, Hutter's optimally intelligent agent (Hutter 2004), nor is it highly specialized in the sense of current AI software. It has strong general capability, yet is biased toward the class of environments in which human intelligence develops - a class of environments whose detailed formalization remains largely an unsolved problem.

\section{The Information Processing Perspective}

Much of modern cognitive science uses the computer as a metaphor for the mind. This perspective differs from the system-theoretic ideas of Piaget and Vygotsky, but at the same time provides a more direct mapping to the target implementation for AGI systems.

According to the information-processing perspective, cognitive development in infancy and 


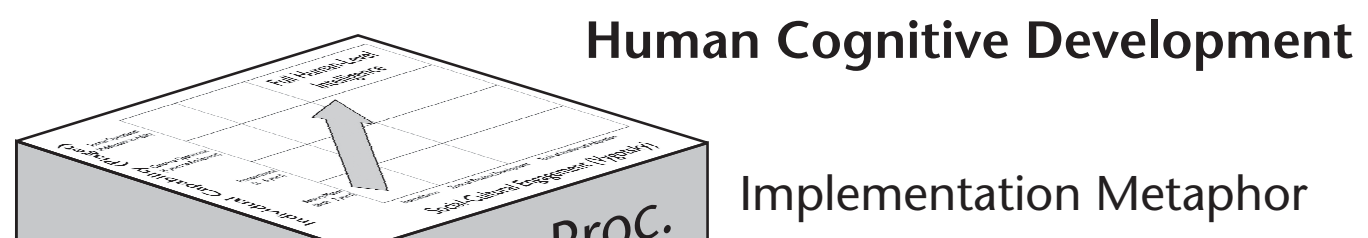

Information Theory

Existing Realization

Figure 4. The Substrata for the AGI Landscape.

childhood is due to changes in both hardware (neural maturation, for example, synaptogenesis, neural pruning, and others) and software (for example, acquisition of strategies for acquiring and processing information). Rather than advocating a stage-based approach, this perspective often highlights processes of change that are gradual or continuous. For example, short-term memory - typically measured by the ability to remember a string of random letters or digits - improves linearly during early childhood (Dempster 1981).

\section{Challenge: Finding Tasks and Environments}

The next challenge we address is that of defining appropriate tasks and environments for assessing progress in AGI where all of the Laird and Wray characteristics are active, and thus all of the requirements must be confronted. The usefulness of any task or environment for this purpose is critically dependent on how well it provides a basis for comparison between alternative approaches and architectures. With this in mind, we believe that both tasks and environments must be designed or specified with some knowledge of each other. For example, consider the competition to develop an automobile capable of driving itself across a roughly specified desert course. ${ }^{1}$ While much of the architecture and implementation would likely be useful for the later competition for autonomous city driving, ${ }^{2}$ such a different environment required significant reconsideration of the tasks and subtasks themselves.
Tasks, therefore, require a context to be useful, and environments, including the AGI's embodiment itself, must be considered in tandem with the task definition. Using this reasoning, we decided to define scenarios that combine both tasks and their necessary environments.

Further, since we are considering multiple scenarios as well as multiple approaches and architectures, it is also important to be able to compare and contrast tasks belonging to different scenarios. With this in mind, we chose to proceed by first articulating a rough heuristic list of human intelligence competencies (figure 5). As a rule of thumb, tasks may be conceived as ways to assess competencies within environments. However, contemporary cognitive science does not give us adequate guidance to formulate anything close to a complete, rigid, and refined list of competencies. What we present here must be frankly classified as an intuitive approach for thinking about task generation, rather than a rigorous analytical methodology from which tasks can be derived.

\section{Environments and Embodiments for AGI}

General intelligence in humans develops within the context of a human body, complete with many thousands of input sensors and output effectors, which is itself situated within the context of a richly reactive environment, complete with many other humans, some of which may be caregivers, teachers, collaborators, or adversaries. Human perceptual input ranges from skin sensors reacting to temperature and pressure, smell and taste sensors in the nose and mouth, sound and light sensors in the ears and eyes, to internal proprioceptive sen- 


\begin{tabular}{|c|c|c|c|c|c|}
\hline Perception & Memory & Attention & $\begin{array}{c}\text { Social } \\
\text { Interaction }\end{array}$ & Planning & Motivation \\
\hline Vision & Working & Visual & Communication & Tactical & Subgoal creation \\
\hline Smell & Episodic & Auditory & Appropriateness & Strategic & Affect-based \\
\hline Touch & Implicit & Social & Social Inference & Physical & $\begin{array}{c}\text { Deferred } \\
\text { Gratification }\end{array}$ \\
\hline Taste & Semantic & Behavioral & Cooperation & Social & Altruism \\
\hline Audition & Procedural & & Competition & & \\
\hline Cross-modal & & Communication & Relationships & & $\begin{array}{l}\text { Modeling Self / } \\
\text { Other }\end{array}$ \\
\hline Proprioceptive & Reasoning & Gestural & & Emotion & Self-Awareness \\
\hline & Induction & Verbal & Learning & Perceived & $\begin{array}{l}\text { Other- } \\
\text { Awareness }\end{array}$ \\
\hline Actuation & Deduction & Musical & Imitation & Expressed & Relationships \\
\hline Physical Skills & Abduction & Pictorial & Reinforcement & Control & Self-Control \\
\hline Tool Use & Physical & Diagrammatical & Dialogical & Understanding & Theory of Mind \\
\hline Navigation & Causal & $\begin{array}{l}\text { Language } \\
\text { Acquisition }\end{array}$ & Media-Oriented & Sympathy & Sympathy \\
\hline Proprioceptive & Associational & Cross-Modal & Experimentation & Empathy & Empathy \\
\hline & \multicolumn{2}{|c|}{ Building/Creation } & \multicolumn{3}{|c|}{ Quantitative } \\
\hline & \multirow{3}{*}{\multicolumn{2}{|c|}{$\begin{array}{l}\text { Physical Construction with Objects } \\
\text { Formation of Novel Concepts } \\
\text { Verbal Invention }\end{array}$}} & \multicolumn{3}{|c|}{ Counting Observed Entities } \\
\hline & & & \multicolumn{3}{|c|}{ Grounded Small Number Arithmetic } \\
\hline & & & \multicolumn{3}{|c|}{$\begin{array}{c}\text { Comparison of Quantitative Properties } \\
\text { of Observed Entities }\end{array}$} \\
\hline & \multicolumn{2}{|c|}{ Social Organization } & \multicolumn{3}{|c|}{ Measurement Using Simple Tools } \\
\hline
\end{tabular}

Figure 5. Some of the Important Competency Areas Associated with Human-Level General Intelligence.

sors that provide information on gravitational direction and body joint orientation, among others. Human output effectors include the many ways we can affect the physical states of our environment, like producing body movements through muscles, sound waves using body motions, or producing vibrations through vocal cords, for example. Effectors like the muscles controlling gaze direction and focus of the eyes also directly affect environmental sensing.

One of the distinctive challenges of achieving AGI is thus how to account for a comparably rich sensory/motor experience. The history of AI is replete with various attempts at robotic embodiment, from Gray Walter's Tortoise (Walter 1953) through Honda's ASIMO. Virtual embodiment has also been utilized, exploiting software systems that simulate bodies in virtual environments, such as Steve Grand's Creatures (Grand 2001).

There are many challenges in determining an appropriate level of body sophistication and environmental richness in designing either physical or virtual bodies and environments. Regrettably, nearly every AI or AGI project that has included situated embodiment has done so in some unique, incompatible and typically nonrepeatable form. Various leagues of the RoboCup competition (Kitano 1997) have been a notable exception to this, where a commercially available hardware robotic platform such as the Sony AIBO or Aldebaran Nao is specified, with contestants only allowed to customize the software of the robotic players. Unfortunately, the limited nature of the RoboCup task - a modified game of soccer - is too narrow to be suitable as a benchmark for AGI.

\section{The Breadth of Human Competencies}

Regardless of the particular environment or embodiment chosen, there are, intuitively speaking, certain competencies that a system must possess to be considered a full-fledged AGI. For instance, even if an AI system could answer questions involving previously known entities and concepts vastly more intelligently than IBM's Watson, we would be reluctant to classify it as human-level if it could not create new concepts when the situation called for it.

While articulation of a precise list of the competencies characterizing human-level general intelligence is beyond the scope of current science (and 
definitely beyond the scope of this article), it is nevertheless useful to explore the space of competencies, with a goal of providing heuristic guidance for the generation of tasks for AGI systems acting in environments. Figure 5 lists a broad set of competencies that we explored at the road-mapping workshop: 14 high-level competency areas, with a few key competency subareas corresponding to each. A thorough discussion of any one of these subareas would involve a lengthy review paper and dozens of references, but for the purposes of this article an evocative list will suffice.

We consider it important to think about competencies in this manner - because otherwise it is too easy to pair a testing environment with an overly limited set of tasks biased to the limitations of that environment. What we are advocating is not any particular competency list, but rather the approach of exploring a diverse range of competency areas, and then generating tasks that evaluate the manifestation of one or more articulated competency areas within specified environments.

Some of the competencies listed may appear intuitively simpler and earlier stage than others, at least by reference to human cognitive development. The developmental theories reviewed earlier in this article each contain their own hypotheses regarding the order in which developing humans achieve various competencies. However, after careful consideration we have concluded that the ordering of competencies isn't necessary for an AGI capability roadmap. In many cases, different existing AGI approaches pursue the same sets of competencies in radically different orders, so imposing any particular ordering on competencies would bias the road-mapping effort toward particular AGI approaches, something antithetical to the collaboration and cooperation sorely needed in the present stage of AGI development.

For example, developmental robotics (Lungarella et al. 2003) and deep learning (Bengio 2009) AGI approaches often assume that perceptual and motor competencies should be developed prior to linguistic and inferential ones. On the other hand, logic-focused AGI approaches are often more naturally pursued by beginning with linguistic and inferential competencies, and only afterwards moving to perception and actuation in rich environments. By dealing with scenarios, competencies, and tasks, but not specifying an ordering of competencies or tasks, we are able to present a capability roadmap that is friendly to existing approaches and open to new approaches as they develop.

\section{Supporting Diverse Concurrent Research Efforts}

Balancing the AGI community's need for collaborative progress while still supporting a wide range of independent research and development efforts, we must allow for concurrent activity in many regions of the landscape. This requires that many of the unsolved lower-level aspects of general intelligence such as visual perception and fine-motor control be finessed in some of our scenarios, replaced by an approximate high-level implementation or even a human surrogate, until we have sufficiently rich underlying systems as a common base for our research. It is sometimes argued that since human intelligence emerges and develops within a complex body richly endowed with innate capabilities, AGI may only be achieved by following the same path in a similar embodiment. But until we have the understanding and capability to construct such a system, our best hope for progress is to foster concurrent but ultimately collaborative work.

\section{Scenarios for Assessing AGI}

In order to create a roadmap toward human-level AGI, one must begin with one or more particular scenarios. By a scenario we mean a combination of tasks to be performed within a specified environment, together with a set of assumptions pertaining to the way the AGI system interacts with the environment along with the inanimate entities and other agents therein. Given a scenario as a basis, one can then talk about particular subtasks, their ordering and performance evaluation.

A wide variety of scenarios may be posited as relevant to AGI; here we review seven that were identified by one or more participants in the AGI Roadmap Workshop as being particularly interesting. Each of these scenarios may be described in much more detail than we have done here; this section constitutes a high-level overview, and more details on several of the scenarios is provided in the references.

\section{General Video-Game Learning}

This scenario addresses many challenges at once: providing a standard, easily accessible, situated embodiment for AGI research; a gradient of ever increasing sensory/motor requirements to support gradual development, measurement, and comparison of AGI capabilities; a wide range of differing environments to test generality of learning ability; and a compelling demonstration of an AGI's capability or lack thereof, easily evaluated by nonspecialists, based on widely common human experiences playing various video games.

The goal of this scenario would not be humanlevel performance of any single video game, but the ability to learn and succeed at a wide range of video games, including new games unknown to the AGI developers before the competition. The contestant system would be limited to a uniform sensory/motor interface to the game, such as video 
and audio output and controller input, and would be blocked from any access to the internal programming or states of the game implementation. To provide for motivation and performance feedback during the game, the normal scoring output would be mapped to a uniform hedonic (pain/pleasure) interface for the contestant. Contestants would have to learn the nature of the game through experimentation and observation, by manipulating game controls and observing the results. The scores achieved against a preprogrammed opponent or story line would provide a standard measure of achievement along with the time taken to learn and win or advance in each kind of game.

The range of video games used for testing in this scenario could be open ended in both simplicity and sophistication. An early game like Pong might itself prove too challenging a starting point, so even simpler games may be selected or developed. Since success at most video games would require some level of visual intelligence, general videogame learning (GVL) would also provide a good test of computer vision techniques, ranging from relatively simple two-dimensional object identification and tracking in Pong to full three-dimensional perception and recognition in a game like Tomb Raider or Half-Life at the highest levels of performance. Various genres of video games such as the early side-scrolling games (for example, Super Mario Brothers) to first person shooters (for example, Doom, Half-Life) to flight simulations (for example, Microsoft Flight Simulator, Star Wars XWing) provide rich niches where different researchers could focus and excel, while the common interface would still allow application of learned skills to other genres.

Among other things, in order to effectively play many videos games a notable degree of strategic thinking must be demonstrated, such as the ability to map situations to actions while considering not just the short-term but also the longer-term implications of choosing an action. Such capability, often associated with the notion of the credit assignment problem, is one that remains to be demonstrated in a scalable way. GVL provides a effective platform for such demonstration.

This scenario has the added benefit of supporting the growth of a research community focused on the specification and development of appropriate tests for AGI. Such a community would create the video game tests, in collaboration with the original game vendors in many cases, and both administer the tests and report the results through an Internet forum similar to the SPEC-style system performance reporting sites. ${ }^{3}$

\section{Preschool Learning}

In the spirit of the popular book All I Really Need to
Know I Learned in Kindergarten (Fulghum 1989), it is appealing to consider early childhood education such as kindergarten or preschool as inspiration for scenarios for teaching and testing AGI systems. The details of this scenario are fleshed out by Goertzel and Bugaj (2009).

This scenario has two obvious variants: a physical preschool-like setting involving a robot, and a virtual-world preschool involving a virtual agent. The goal in such scenarios is not to imitate human child behavior precisely but rather to demonstrate a robot or virtual agent qualitatively displaying similar cognitive behaviors to a young human child. This idea has a long and venerable history in the AI field - Alan Turing's original 1950 paper on AI, where he proposed the famous Turing test, contains the suggestion that "Instead of trying to produce a programme to simulate the adult mind, why not rather try to produce one which simulates the child's?" (Turing 1950).

This childlike cognition-based approach seems promising for many reasons, including its integrative nature: what a young child does involves a combination of perception, actuation, linguistic and pictorial communication, social interaction, conceptual problem solving, and creative imagination. Human intelligence develops in response to the demands of richly interactive environments, and a preschool is specifically designed to be just such an environment with the capability to stimulate diverse mental growth. The richness of the preschool environment suggests that significant value is added by the robotics-based approach; but much could also potentially be achieved by stretching the boundaries of current virtual-world technology.

Another advantage of focusing on childlike cognition is the wide variety of instruments for measuring child intelligence developed by child psychologists. In a preschool context, the AGI system can be presented with variants of tasks typically used to measure the intelligence of young human children.

It doesn't currently make sense to outfit a virtual or robot preschool as a precise imitation of a human preschool - this would be inappropriate since a contemporary robotic or virtual body is rather differently capable than that of a young human child. The aim in constructing an AGI preschool environment should be to emulate the basic diversity and educational character of a typical human preschool.

To imitate the general character of a human preschool, several learning centers should be available in a virtual or robot preschool. The precise architecture will be adapted through experience, but initial learning centers might include the Blocks Center - a table with blocks of various shapes and sizes on it; the Language Center: a circle of chairs, intended for people to sit around and talk with the 
AGI; the Manipulatives Center — with a variety of different objects of different shapes and sizes, intended to teach a wide range of visual and motor skills; the Ball Play Center - where balls are kept in chests and there is space for the AGI to kick or throw the balls around alone or with others; or the Dramatics Center - where the AGI can both observe and enact various movements and roles.

\section{Reading Comprehension}

The next scenario is closely related to the previous one, but doesn't require embodiment in a virtual world, and makes a special commitment as to the type of curriculum involved. In this scenario, an aspiring AGI should work through the grade school reading curriculum, and take and pass the assessments normally used to assess the progress of human children. This obviously requires understanding a natural language (NL) text and being able to answer questions about it. However, it also requires some not so obvious abilities.

Very early readers are usually picture books that tightly integrate the pictures with the text. In some, the story is mostly conveyed through the pictures. In order to understand the story, the pictures must be understood as well as the NL text. This requires recognizing the characters and what the characters are doing. Reference resolution is required between characters and events mentioned in the text and illustrated in the pictures. The actions that the characters are performing must be recognized from snapshot poses, unlike the more usual action recognition from a sequence of frames taken from a video. The next stage of readers are early chapter books, which use pictures to expand on the text. Although the story is now mainly advanced through the text, reference resolution with the pictures is still important for understanding.

Instructors of reading recognize "four roles of a reader as: meaning maker, code breaker, text user, and text critic ... meaning makers read to understand ... comprehension questions [explore] literal comprehension, inferential comprehension, and critical thinking" (Sundance 2001). Code breakers translate written text to speech and vice versa. Text users identify whether the text is fiction or nonfiction. "If the book is factual, they focus on reading for information. If a text is fiction, they read to understand the plot, characters, and message of the story .... Text critics evaluate the author's purpose and the author's decisions about how the information is presented ... check for social and cultural fairness ... look for misinformation ... and think about their own response to the book and whether the book is the best it might be" (Sundance 2001).

The roles of meaning maker, code breaker, and text user are mostly, though not entirely, familiar to people working in natural language under- standing. However, the role of text critic is new, requiring metalevel reasoning about the quality of the text, its author's intentions, the relation of the text to the reader's society and culture, and the reader's own reaction to the text, rather than merely reasoning about the information in the text.

This scenario fulfills most of the Laird and Wray criteria handily, and a few less obviously. The AGI's environment in this scenario is not dynamic in a direct sense (C2), but the AGI does have to reason about a dynamic environment to fulfill the tasks. In a sense, the tasks involved are fixed rather than novel (C5), but they are novel to the AGI as it proceeds through them. Other agents affect task performance (C4) if group exercises are involved in the curriculum (which indeed is sometime the case). Many of the specific abilities needed for this scenario are discussed in the next scenario.

\section{Story or Scene Comprehension}

Focused on scene and story comprehension, this scenario shares aspects of the preschool and reading comprehension scenarios. Like the reading scenario, it focuses on a subset of childlike performance - but a different subset, involving a broader variety of engagements with the world. Scene comprehension here does not mean only illustrations, but real-world scenes, which can be presented at different granularities, media, and complexities (cartoons, movies, or theatrical performances, for instance). This approach differs from the reading scenario in that it more directly provides a dynamic environment. If group exercises are included then all the Laird and Wray criteria are fulfilled in a direct and obvious way.

The story or scene comprehension scenario focuses on the relationship between perception, mental representation, and natural language. Here, the system might be presented with a story, which it needs to analyze semantically and then re-present in a different form (for instance, as a movie sequence, or another kind of retelling). In the other direction, the system is shown a movie or cartoon sequence, which it needs to comprehend and re-present using language. This approach lends itself to a variety of standardizable test scenarios, which allow the direct comparison of competing AGI architectures with each other as well as with child performance using identical input.

Note that these tests ignore the interaction of the system and the environment, and the particular needs of the system itself. It is often argued that general intelligence might only be attained by a motivated, interacting system, but this scenario would allow the comparison of motivated, interactive architectures with less comprehensive processing-oriented architectures.

AGI system capabilities in story and scene comprehension are probably not independent but relat- 
ed, since both tasks focus on modeling the relationships between perception, language, and thought, which are not unidirectional. Further tasks might focus on the interplay between these areas, by combining expression and comprehension. This could either be done in a fully interactive manner (that is, by designing a system that has to respond to a teacher), or self-interactive (two agents learn to communicate with each other, while sharing an environment). For an example design of such a self-interactive scenario, see Steels (2010).

\section{School Learning}

The virtual school student scenario continues the virtual preschool scenario but is focused on higher cognitive abilities assuming that, if necessary, lower-level skills will be finessed. In particular, it is assumed that all interfaces with the agent are implemented at a symbolic level: the agent is not required to process a video stream, to recognize speech and gestures, to balance its body and avoid obstacles while moving in space, and others. All these can be added later to enhance the challenge, but a key feature of this scenario is that they can also be finessed, allowing for early exploration of such capabilities without waiting for other system developments. On the other hand, it is critically important in the scenario for the agent to make academic progress at a human student level, to understand human minds, and to understand and use classroom-related social relations in the environment in which it is embedded.

In this scenario, the agent is embedded in a real high school classroom by means of a virtual-reality-based interface. The agent lives in a symbolic virtual world that is continuously displayed on a big screen in the classroom. The virtual world includes a virtual classroom represented at a symbolic (object) level, including the human instructor and human students represented by simplistic avatars. The agent itself is represented by an avatar in this virtual classroom. The symbolic virtual world is synchronized with the real physical world with the assistance of intelligent monitoring and recording equipment performing scene analysis, speech recognition, language comprehension, gesture recognition, and others (if necessary, some or all of these functions will be performed by hidden human personnel running the test; students should not be aware of their existence). The study material, including the textbook and other curriculum materials available to each student, will be encoded electronically and made available to the agent at a symbolic level.

A particularly challenging aspect of this scenario is that the agent will be evaluated not only on its own learning and problem-solving performance, but also on its approach to problem solving, based on its interactions with students and with the instructor. Here the general metrics for self-regulated learning will be used (Winne and Perry 2000). In addition, social performance of the agent can be evaluated based on surveys of students and standard psychological metrics. Another potentially important measure will be the effect of the agent presence in the classroom on human student learning.

\section{The Wozniak Test}

In an interview a few years ago, Steve Wozniak of Apple Computer fame expressed doubt that there would ever be a robot that could walk into an unfamiliar house and make a cup of coffee (Wozniak and Moon 2007). We feel that this task is demanding enough to stand as a Turing test equivalent for embodied AGI. Note that the Wozniak test is a single, special case of Nils Nilsson's general employment test for human-level AI (Nilsson 2005).

A robot is placed at the door of a typical house or apartment. It must find a doorbell or knocker, or simply knock on the door. When the door is answered, it must explain itself to the householder and enter once it has been invited in. (We will assume that the householder has agreed to allow the test in his or her house, but is otherwise completely unconnected with the team doing the experiment, and indeed has no special knowledge of AI or robotics at all.) The robot must enter the house, find the kitchen, locate local coffee-making supplies and equipment, make coffee to the householder's taste, and serve it in some other room. It is allowed, indeed required by some of the specifics, for the robot to ask questions of the householder, but it may not be physically assisted in any way.

The current state of the art in robotics falls short of this capability in a number of ways. The robot will need to use vision to navigate, identify objects, possibly identify gestures ("the coffee's in that cabinet over there"), and to coordinate complex manipulations. Manipulation and physical modeling in a tight feedback learning loop may be necessary, for example, to pour coffee from an unfamiliar pot into an unfamiliar cup. Speech recognition and natural language understanding and generation will be necessary. Planning must be done at a host of levels ranging from manipulator paths to coffee-brewing sequences.

The major AGI advance for a coffee-making robot is that all of these capabilities must be coordinated and used appropriately and coherently in aid of the overall goal. The usual setup, task definition, and so forth are gone from standard narrow AI formulations of problems in all these areas; the robot has to find the problems as well as to solve them. That makes coffee-making in an unfamiliar house a strenuous test of a system's adaptivity and ability to deploy common sense.

Although standard shortcuts might be used, 


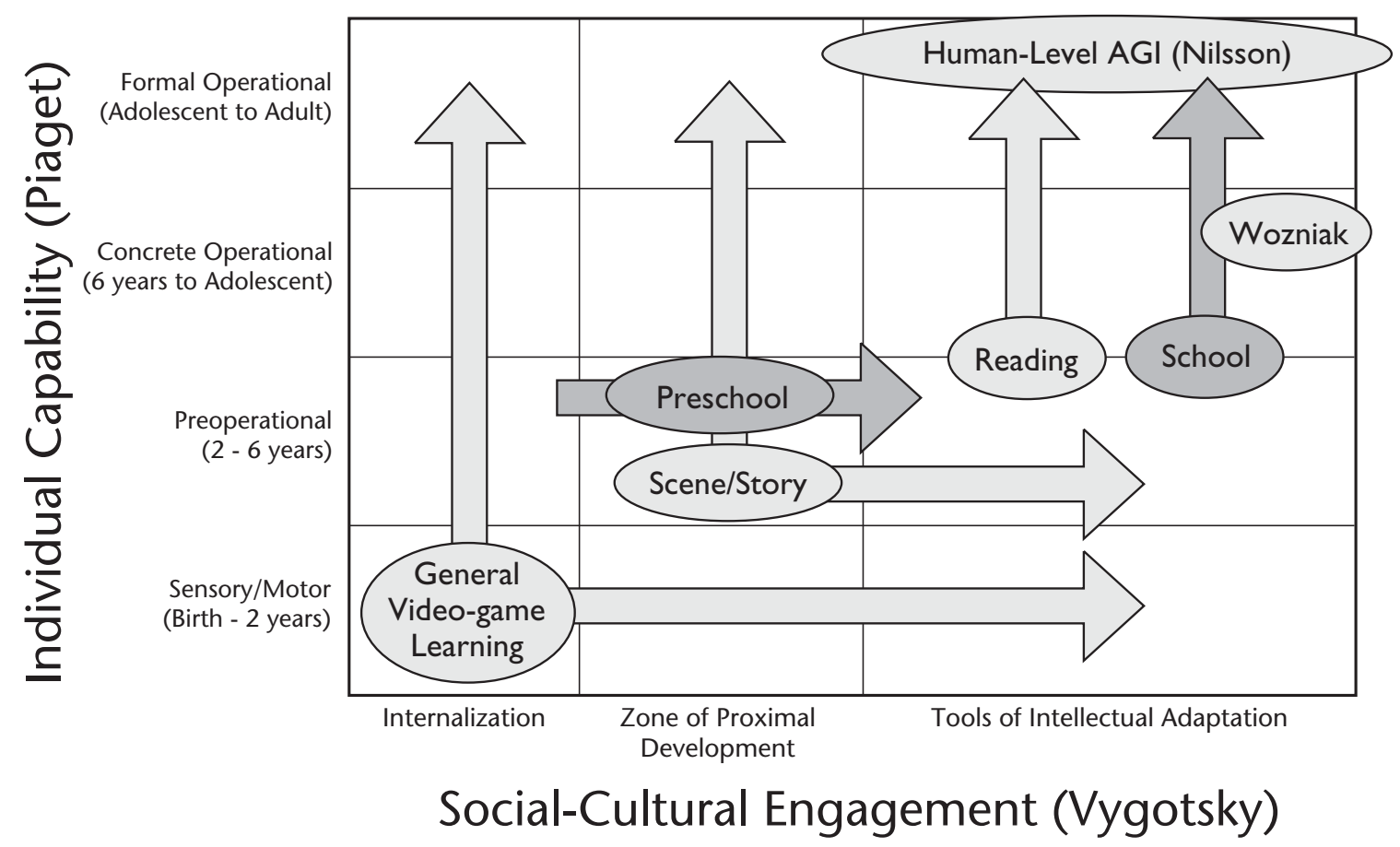

Figure 6. Scenario Milestones on the AGI Landscape.

such as having a database of every manufactured coffeemaker built in, it would be prohibitive to have the actual manipulation sequences for each one preprogrammed, especially given the variability in workspace geometry, dispensers and containers of coffee grounds, and so forth. Transfer learning, generalization, reasoning by analogy, and in particular learning from example and practice are almost certain to be necessary for the system to be practical.

Coffee-making is a task that most 10-year-old humans can do reliably with a modicum of experience. A week's worth of being shown and practicing coffee making in a variety of homes with a variety of methods would provide the grounding for enough generality that a 10 year old could make coffee in the vast majority of homes in a Wozniak test. Another advantage to this test is it would be extremely difficult to cheat, since the only reasonably economical way to approach the task would be to build general-learning skills and have a robot that is capable of learning not only to make coffee but any similar domestic chore.

\section{An Initial Road Map for Achieving AGI}

Having set forth these seven scenarios, we can now proceed to array them across the previously defined AGI landscape (figure 3). Figure 6 shows the result of this mapping, with some scenarios like General Video Game Learning and Scene/Story Comprehension spanning large areas of the landscape as their content increases in complexity and cognitive challenge. Just as we consider the Laird and Wray requirements as a practical starting point and our scenarios as initial representatives of a much larger, more representative collection, we hope this initial road map is enhanced and corrected as our collective experience in AGI grows.

\section{Remaining Whitespace}

In our view, the suggested scenarios represent a significant step in populating the AGI Landscape, but many alternative scenarios are conceivable, and might even cover areas that we have incompletely addressed or not mentioned at all.

Among the areas that might need more attention are aesthetic appreciation and performance, structured social interaction, and skills that require high cognitive function and integration.

Aesthetic appreciation and performance include composition, literature, artistic portrayal, dance, and others. Examining the appreciation of aesthetics is bound to increase our understanding of the motivational system, of mental representation, 
and of metacognition. Aesthetic performance adds manipulation, creation, and adaptation of physical and imagined objects, theory of mind, aspects of sociality, and many things more.

Structured social interaction includes goal adoption, collaboration, competition and exploitation, negotiation, discourse and joint decision making, group organization, leadership, and so on. Scenarios in the social domain will require representations and assessment of social setups, the mental states of individuals, and possible ramifications for the agent's own goals.

Skills that require high cognitive function and integration include, for instance, complex rescue scenarios, physical shopping, and skilled human assistance. Each of these task areas would require complex coordination between interaction, exploration, evaluation, and planning.

Thus, we encourage and invite suggestions for additional scenarios, as long as these are firmly rooted in an AGI approach, that is, each scenario should foster our understanding of general, humanlike intelligence, and not simply provide narrow engineering solutions to a limited task.

\section{From Scenarios to Tasks, Metrics, and Challenges}

The description of scenarios and competency areas, as we have presented here, makes it possible to articulate specific tasks for assessing progress toward AGI in a principled manner. For each of the scenarios reviewed above (or other analogous scenarios), one may define a specific task-set, where each task addresses one or more of the competency areas in the context of the scenario. To constitute a reasonable approximation of an AGI test suite or overall AGI challenge, the total set of tasks for a scenario must cover all the competency areas. Each task must also be associated with some particular performance metric - quantitative wherever possible, but perhaps qualitative in some cases depending on the nature of the task.

The obvious risk of an AGI evaluation approach based on a long list of tasks is that it is susceptible to solution by the so-called "big switch statement" approach, in which separate narrowly specialized programs corresponding to the individual tasks are combined together in a simplistic harness. Some may believe that human intelligence is well approximated by a big switch statement of this sort, but we take a different perspective, which doesn't rule out the possibility, but also doesn't focus on or encourage this approach.

In addition, the competency areas alluded to above include many that focus on learning and generalization. So even if a big switch statement approach is used to handle tasks based on these requirements, the switch will be choosing between a large number of specialized programs that in themselves could handle a great deal of learning.

\section{Example Tasks}

For sake of brief illustration, table 1 roughly describes a handful of example tasks and task families corresponding to a few of the scenarios described above. Much of the work of fleshing out the initial roadmap described here will involve systematically creating a substantial list of tasks corresponding to the scenarios chosen, using an exploratory enumeration of human general intelligence competencies as a guide (perhaps a refined version of the list in figure 5). Each task will then need to be associated with quantitative performance metrics.

Some of the examples in table 1 are highly specific, others are broader task families. Our feeling is that it will generally be better to identify moderately broad task families rather than highly specific tasks, in order to encourage the creation of systems with more robust and flexible functionality.

\section{Multidimensional Challenges}

What then is the right sort of challenge to present to a would-be AGI system? We suggest the following: to address a few dozen closely interrelated, moderately broadly defined tasks in an environment drawn from a moderately broad class (for example, an arbitrary preschool or textbook rather than specific ones determined in advance). If the environment is suitably rich and the tasks are drawn to reflect the spectrum of human general intelligence competencies, then this sort of challenge will motivate the development of genuine human-level AGI systems.

This sort of challenge is not nearly as well specified as a chess contest, RoboCup, or the DARPA Grand Challenge, but we feel the complexity and heterogeneity here is directly reflective of the complexity and heterogeneity of human general intelligence. Some have posited that there are simple core principles underlying all human-level general intelligence. Even if this turns out to be true, the real-world manifestation of these principles what can actually be measured - is highly complex and involves multiple interrelated competencies defined through interaction with a rich, dynamic world.

\section{Challenges and Competitions}

Finally, we emphasize that our main goal here is to propose a capability roadmap for the AGI research community, not a competition or contest. Competitions can be valuable, and we would be happy to see one or more developed along the lines of the capability roadmap we have described. However, we doubt any successful competition could ever cover the full extent of a roadmap such as this. 


\begin{tabular}{|c|c|c|c|}
\hline Scenario & $\begin{array}{l}\text { Competency } \\
\text { Area }\end{array}$ & Subarea & Example Task or Task Family \\
\hline $\begin{array}{l}\text { Virtual } \\
\text { Preschool }\end{array}$ & Learning & Dialogical & $\begin{array}{l}\text { Learn to build a particular structure of blocks (such as a pyramid) faster based on a } \\
\text { combination of imitation, reinforcement, and verbal instruction, than by imitation } \\
\text { and reinforcement without verbal instruction }\end{array}$ \\
\hline $\begin{array}{l}\text { Virtual } \\
\text { Preschool }\end{array}$ & $\begin{array}{l}\text { Modeling Self } \\
\text { and Other }\end{array}$ & $\begin{array}{l}\text { Theory of } \\
\text { Mind }\end{array}$ & $\begin{array}{l}\text { While Sam is in the room, Ben puts the red ball in the red box. Then Sam leaves } \\
\text { and Ben moves the red ball to the blue box. Sam returns and Ben asks him where } \\
\text { the red ball is. The agent is asked where Sam thinks the ball is. }\end{array}$ \\
\hline $\begin{array}{l}\text { Virtual } \\
\text { School } \\
\text { Student }\end{array}$ & Learning & $\begin{array}{l}\text { Media- } \\
\text { oriented }\end{array}$ & $\begin{array}{l}\text { Starting from initially available basic concepts (a number, a variable, a function), } \\
\text { demonstrate academic progress in learning how to solve problems from the text- } \\
\text { book using techniques described in the same textbook. The agent should move step } \\
\text { by step, from simple to advanced problems, from one domain to another. }\end{array}$ \\
\hline $\begin{array}{l}\text { Robot } \\
\text { Preschool }\end{array}$ & Actuation & $\begin{array}{l}\text { Propriocep- } \\
\text { tion }\end{array}$ & $\begin{array}{l}\text { The teacher moves the robot's body into a certain configuration. The robot is asked } \\
\text { to restore its body to an ordinary standing position, and then repeat the configura- } \\
\text { tion that the teacher moved it into. }\end{array}$ \\
\hline $\begin{array}{l}\text { Robot } \\
\text { Preschool }\end{array}$ & Memory & Episodic & $\begin{array}{l}\text { Ask the robot about events that occurred at times when it received a particularly } \\
\text { large, or particularly small, reward for its actions; it should be able to answer simple } \\
\text { questions about these significant events, with significantly more accuracy than } \\
\text { about events occurring at random times }\end{array}$ \\
\hline $\begin{array}{l}\text { Wozniak } \\
\text { Test }\end{array}$ & Communication & Gestural & $\begin{array}{l}\text { The robot will be directed to the kitchen. It must understand gestures indicating } \\
\text { that it should follow an indicated path, or know how to follow its guide, and know } \\
\text { when either is appropriate. }\end{array}$ \\
\hline $\begin{array}{l}\text { Wozniak } \\
\text { Test }\end{array}$ & Actuation & Navigation & The robot must complete its task without colliding people, walls, furniture, or pets. \\
\hline $\begin{array}{l}\text { Wozniak } \\
\text { Test }\end{array}$ & $\begin{array}{l}\text { Social } \\
\text { Interaction }\end{array}$ & behavior & $\begin{array}{l}\text { The robot must recognize and appropriately respond to the situation where it has } \\
\text { knocked on the wrong door, the householder is not home, or the robot is not wel- } \\
\text { come to enter the house. }\end{array}$ \\
\hline $\begin{array}{l}\text { Wozniak } \\
\text { Test }\end{array}$ & Reasoning & Physical & $\begin{array}{l}\text { Be able to use incomplete or alternative tools and equipment. For instance, a drip } \\
\text { pot may be used without its top, but not a percolator. This may require physical } \\
\text { simulation, based on an understanding of naive physics. }\end{array}$ \\
\hline $\begin{array}{l}\text { Wozniak } \\
\text { Test }\end{array}$ & Reasoning & Induction & $\begin{array}{l}\text { On the other hand, the above-mentioned knowledge about drip pots and percola- } \\
\text { tors may be gathered through inductive reasoning based on observations in multi- } \\
\text { ple relevant situations. }\end{array}$ \\
\hline
\end{tabular}

Table 1. Mapping Human General Intelligence Competencies to Scenario Tasks and Task Families.

One reason for this is that a competition is very likely to involve concrete decisions regarding the prioritization or temporal ordering of tasks, which will inevitably result in the competition having widely differential appeal to AGI researchers depending on their chosen scientific approach.

The primary value to be derived through wide adoption of a capability roadmap of this nature is the concentration of multiple researchers pursuing diverse approaches on common scenarios and tasks - which allows them to share ideas and learn from each other much more than would be the case otherwise. There is much to be gained through multiple researchers pursuing the same concrete challenges in parallel, with or without the presence of an explicit judged competition.

\section{Call For Collaboration}

We have explained our vision for the creation of a roadmap to human-level artificial general intelligence, selected goals and starting points, established a landscape, populated it with a number of diverse scenarios, and fleshed out some of the details. What's next is to put more meat on the bones: describe the scenarios in more detail, refine the list of specific competency areas, and then create tasks and metrics along the lines outlined above. Once this is done, we will have a capability roadmap clearly articulating some plausible pathways to AGI; and we will have the basis for creating holistic, complex challenges that can meaningfully assess progress on the AGI problem.

Such a roadmap does not give a highly rigorous, objective way of assessing the percentage of progress toward the end goal of AGI. However, it gives a much better sense of progress than one would have otherwise. For instance, if an AGI system performed well on diverse metrics corresponding to tasks assessing 50 percent of the competency areas listed above, in several of the above scenarios, the creators of this system would seem justified in claiming to have made very substantial progress toward AGI. If the number were 90 percent rather than 50 percent, one would seem justified in claiming to be almost there. If the number were 25 percent, this would give one a reasonable claim to interesting AGI progress. This kind of qualitative assessment of progress is not the most one could hope for, but it is better than the 
progress indications one could get without this sort of roadmap.

We have already explicitly noted many of the limitations of our treatment of this challenging topic. While there is no consensus among AGI researchers on the definition of general intelligence, we can generally agree on a pragmatic goal. The diversity of scenarios presented reflects a diversity of perspectives among AGI researchers regarding which environments and tasks best address the most critical aspects of AGI. Most likely neither the tentative list of competencies nor the Laird and Wray criteria are either necessary or sufficient. There is no obvious way to formulate a precise measure of progress toward AGI based on the competencies and scenarios provided - though one can use these to motivate potentially useful approximative measures.

But in spite of its acknowledged limitations, a capability roadmap of the sort outlined here allows multiple researchers following diverse approaches to compare their work in a meaningful way; allows researchers, and other observers, to roughly assess the degree of research progress toward the end goal of human-level artificial general intelligence; and allows work on related road-mapping aspects, such as tools roadmaps and study of social implications and potential future applications, to proceed in a more structured way.

With this in mind, we encourage AGI researchers to join with us in the ongoing collaborative construction of a more detailed AGI roadmap along the lines suggested here.

\section{Notes}

1. See the DARPA Grand Challenge Rulebook (www.darpa.mil/grandchallenge05/Rules_8oct04.pdf).

2. See the DARPA Urban Challenge Rules (www. darpa.mil/grandchallenge/rules.asp).

3. See the Standard Performance Evaluation Corporation (www.spec.org).

\section{References}

Arel, I. 2009. Working Toward Pragmatic Convergence: AGI Axioms and a Unified Roadmap. Paper presented at the Workshop on the Future of AGI, Second Conference on Artificial General Intelligence (AGI-09).

Arel, I., and Livingston, S. 2009. Beyond the Turing Test. IEEE Computer 42(3): 90-91.

Bengio, Y. 2009. Learning Deep Architectures for AI. Foundations and Trends in Machine Learning 2(1): 1-127.

Campbell, M.; Hoane, A. J., Jr.; and Hsu, F. 2002. Deep Blue. Artificial Intelligence 134(1-2) (January): 57-83.

Dempster, F. N. 1981. Memory Span: Sources of Individual and Developmental Differences. Psychological Bulletin 89(1): 63-100.

Ferrucci, D. 2010. Build Watson: An Overview of DeepQA for the Jeopardy! Challenge. In Proceedings of the 19th International Conference on Parallel Architectures and Com- pilation Techniques (PACT '10), 1-2. New York: Association for Computing Machinery.

Fulghum, R. 1989. All I Really Need to Know I Learned in Kindergarten. New York: Random House/Ivy Books.

Gardner, H. 1999. Intelligence Reframed: Multiple Intelligences for the 21st Century. New York: Basic Books.

Goertzel, B. 2010. Toward a Formal Definition of RealWorld General Intelligence. In Proceedings of the Third Conference on Artificial General Intelligence (AGI-10). Paris: Atlantis Press.

Goertzel, B., and Bugaj, S. V. 2009. AGI Preschool. In Proceedings of the Second Conference on Artificial General Intelligence (AGI-09). Paris: Atlantis Press.

Grand, S. 2001. Creation: Life and How to Make It. London: Phoenix Books.

Gregory, R. 1996. Psychological Testing: History, Principles, and Applications. London: Allyn and Bacon.

Hutter, M. 2004. Universal Artificial Intelligence: Sequential Decisions Based on Algorithmic Probability. Berlin: Springer. Kitano, H. 1997. RoboCup: The Robot World Cup Initiative. In Proceedings of the First International Conference on Autonomous Agent (Agents-97). New York: Association for Computing Machinery.

Laird, J., and Wray, R. 2010. Cognitive Architecture Requirements for Achieving AGI. In Proceedings of the Third Conference on Artificial General Intelligence (AGI-10). Paris: Atlantis Press.

Laird, J.; Wray, R.; Marinier, R.; and Langley, P. 2009. Claims and Challenges in Evaluating Human-Level Intelligent Systems. In Proceedings of the Second Conference on Artificial General Intelligence (AGI-09). Paris: Atlantis Press.

Legg, S., and Hutter, M. 2007. A Definition of Machine Intelligence. Minds and Machines 17(4) 391-444.

Lungarella, M.; Metta, G.; Pfeifer, R.; and Sandini, G. 2003. Developmental Robotics: A Survey. Connection Science 15(4): 151-190.

Nilsson, N. J. 2005. Human-Level Artificial Intelligence? Be Serious! AI Magazine 26(4): 68-75.

Piaget, J. 1953. The Origins of Intelligence in Children. London: Routledge and Kegan Paul.

Samsonovich, A. V.; Ascoli, G. A.; and De Jong, K. A. 2006. Human-Level Psychometrics for Cognitive Aarchitectures. Paper presented at the Fifth International Conference on Development and Learning (ICDL5), Bloomington, IN, 31 May-3 June.

Steels, L. 2010. Modeling the Formation of Language in Embodied Agents: Methods and Open Challenges. In Evolution of Communication and Language in Embodied Agents, 223-233. Berlin: Springer Verlag.

Sundance 2001. Teacher Guide for Just Kids. Marlborough, MA: Sundance Publishing.

Turing, A. M. 1950. Computing Machinery and Intelligence. Mind 59(236) (October): 433-60.

Vygotsky, L. 1986. Thought and Language. Cambridge, MA: The MIT Press.

Walter, W. G. 1953. The Living Brain. New York: W. W. Norton.

Winne, P. H., and Perry, N. E. 2000. Measuring Self-Regulated Learning, 531-566. Boston: Academic Press.

Wozniak, S., and Moon, P. 2007. Three Minutes with Steve Wozniak. PC World July 19. 
Sam S. Adams (ssadams@us.ibm.com) currently works for IBM Research and was appointed one of IBM's first distinguished engineers in 1996. His far-ranging contributions include founding IBM's first object technology practice, authoring IBM's XML technical strategy, originating the concept of service-oriented architecture, pioneering work in self-configuring and autonomic systems, artificial general intelligence, end-user mashup programming, massively parallel many-core programming, petascale analytics, and data-centered systems. He is a founding member of the AGI community and continues to research bootstrapping human-level general intelligence through the Joshua Blue project.

Itamar Arel (itamar@ece.utk.edu) is an associate professor of electrical engineering and computer science and director of the Machine Intelligence Laboratory at the University of Tennessee. His research focus is on highperformance machine learning architectures and algorithms, with emphasis on deep learning architectures, reinforcement learning, and decision making under uncertainty. Arel is a recipient of the U.S. Department of Energy Early Career Principal Investigator (CAREER) award in 2004. He holds B.S., M.S., and Ph.D. degrees in electrical and computer engineering and an M.B.A. degree, all from Ben-Gurion University in Israel.

Joscha Bach (joscha.bach@hu-berlin.de) studied computer science and philosophy at Berlin's Humboldt University and at Waikato University (NZ). He received a Ph.D. in cognitive science from the University of Osnabrück. His main research interest concerns the design of cognitive architectures, especially with respect to representation, motivation, and affect. He is a research fellow at the Berlin School of Mind and Brain, at Humboldt University of Berlin.

Robert Coop (rcoop@utk.edu) is a doctoral student at the University of Tennessee at Knoxville. He is currently studying machine learning, with a focus on generalized pattern extraction methods.

Rod Furlan (rod.furlan@singularityu.org) is the chief of research and development at Quaternix Research Inc. His work explores the intersection of artificial intelligence, high-performance computing, and quantitative finance. Passionate about technology and a serial autodidact, Furlan is also a Singularity University alumnus and teaching fellow for AI and robotics.

Ben Goertzel (ben@goertzel.org) is chief scientist of the financial prediction firm Aidyia Holdings and chairman of the AI consulting firm Novamente LLC and bioinformatics firm Biomind LLC. He is also leader of the OpenCog open-source artificial general intelligence project, general chair of the AGI conference series, and vice chair of the futurist organization Humanity+. His major research focuses are embodied artificial general intelligence and applications of AI to genomics.

J. Storrs Hall (josh@foresight.org) is an independent Ph.D. scientist and author. His recent book, Beyond AI: Creating the Conscience of the Machine (Prometheus, 2007), was the first full-length treatment of machine ethics. He is active in the machine ethics and AGI research communities, serving on the program committee for the international AGI conference series.
Alexei V. Samsonovich (samsonovich@cos.net) is a research assistant professor at Krasnow Institute for Advanced Study at George Mason University. He holds a Ph.D. in applied mathematics from the University of Arizona, where he codeveloped a continuous-attractor theory of hippocampal spatial maps and a mental state framework for cognitive modeling. His current research focuses on biologically inspired cognitive architectures (BICA). He has chaired annual conferences on BICA since 2008, cofounded and directed the BICA Society since 2010, and edited BICA since 2012.

Matthias Scheutz (mscheutz@indiana.edu) is an associate professor of computer science at Tufts University School of Engineering. His current research interests include agent-based modeling, cognitive modeling, complex cognitive and affective robots for human-robot interaction, computational models of human language processing in mono- and bilinguals, cognitive architectures, distributed agent architectures, and interactions between affect and cognition. Scheutz earned a Ph.D. in philosophy from the University of Vienna in 1995 and a Joint Ph.D. in cognitive science and computer science from the Indiana University Bloomington in 1999.

Matthew Schlesinger (matthews@siu.ude) received his Ph.D. in developmental psychology from the University of California at Berkeley, with postdoctoral research in robotics and machine learning. He is an associate professor of psychology at Southern Illinois University, Carbondale, where he is a member of the Brain and Cognitive Sciences program and directs the Vision Lab. Schlesinger serves as an associate editor of IEEE Transactions on Autonomous Mental Development, in addition to cochairing the TAMD-IEEE Technical Committee. His work integrates a number of approaches that span psychology and machine learning, and includes experimental research with human infants, children, and adults, modeling of perceptual and cognitive processes in embodied agents, as well as neuroimaging.

Stuart C. Shapiro (shapiro@buffalo.edu) is a professor of computer science and engineering and an affiliated professor of linguistics and of philosophy at the University at Buffalo, The State University of New York. He received an SB in mathematics from the Massachusetts Institute of Technology in 1966, and an MS and PhD in computer sciences from the University of Wisconsin - Madison in 1968 and 1971. His primary research interests are in knowledge representation and reasoning, especially in support of natural language competence and cognitive robotics. He is a past chair of ACM/SIGART; a past president of Principles of Knowledge Representation and Reasoning, Incorporated; a life senior member of the IEEE; an ACM Distinguished Scientist; and a Fellow of the AAAI.

John F. Sowa (sowa@bestweb.net) spent 30 years working on research and development projects at IBM and is a cofounder of VivoMind Research, LLC. He has a BS in mathematics from the Massachusetts Institute of Technology, an MA in applied mathematics from Harvard, and a PhD in computer science from the Vrije Universiteit Brussel. He is a fellow of the American Association for Artificial Intelligence. The language of conceptual graphs, which he designed, has been adopted as one of the three principal dialects of the ISO/IEC standard for Common Logic. 


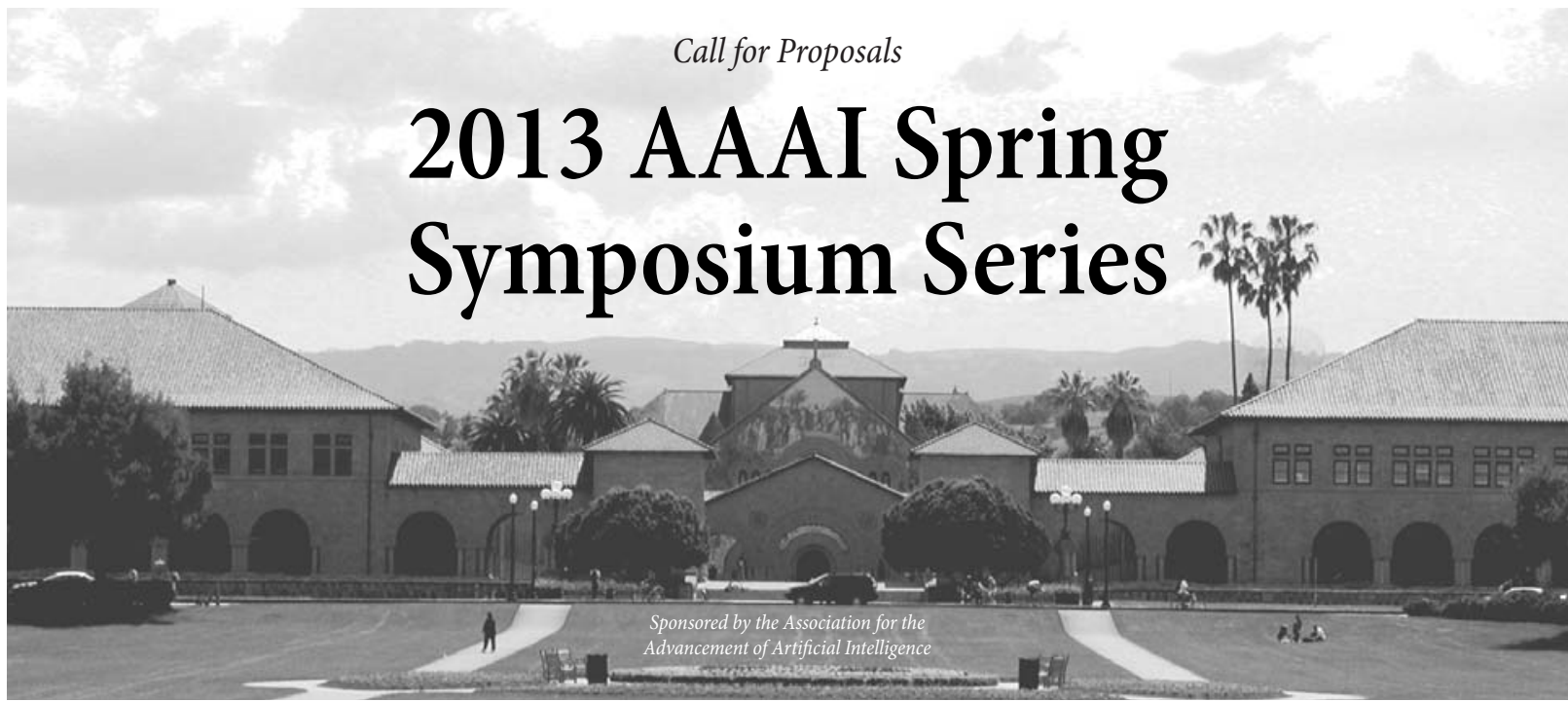

AAAI invites proposals for the 2013 Spring Symposium Series, to be held March 25-27, 2013 at Stanford University, California.

The Spring Symposium Series is an annual set of meetings run in parallel at a common site. It is designed to bring colleagues together in an intimate forum while at the same time providing a significant gathering point for the AI community. The two and one half day format of the series allows participants to devote considerably more time to feedback and discussion than typical one-day workshops. It is an ideal venue for bringing together new communities in emerging fields.

The symposia are intended to encourage presentation of speculative work and work in progress, as well as completed work. Ample time should be scheduled for discussion. Novel programming, including the use of target problems, open-format panels, working groups, or breakout sessions, is encouraged. AAAI Technical Reports will be prepared, and distributed to the participants. Most participants of the symposia will be selected on the basis of statements of interest or abstracts submitted to the symposia chairs; some open registration will be allowed. All symposia are limited in size, and participants will be expected to attend a single symposium.

Proposals for symposia should be between two and five pages in length, and should contain:

so A title for the symposium.

\&o A description of the symposium, identifying specific areas of interest, and general symposium format.

\&o The names and addresses (physical and electronic) of the organizing committee: preferably three or more people at different sites, all of whom have agreed to serve on the committee.

8f A list of potential participants that have been con tacted and that have expressed interest in participating. A common way of gathering potential participants is to send email messages to email lists re- lated to the topic(s) of the symposium. Note that potential participants need not commit to participating, only state that they are interested.

Ideally, the entire organizing committee should collaborate in producing the proposal. If possible, a draft proposal should be sent out to a few of the potential participants and their comments solicited.

Approximately eight symposia on a broad range of topics within and around AI will be selected for the 2013 Spring Symposium Series. All proposals will be reviewed by the AAAI Symposium Committee, (Chair: Matthew E. Taylor, Lafayette College; Cochair: Gita Sukthankar, University of Central Florida; Alan Schultz, Naval Research Laboratory; and Kate Larson, University of Waterloo).

The criteria for acceptance of proposals include:

Perceived interest to the AAAI community. Although AAAI encourages symposia that cross disciplinary boundaries, a symposium must be of interest to some subcommunity of the AAAI membership. Symposia that are of interest to a broad range of AAAI members are also preferred.

Appropriate number of potential participants. Although the series supports a range of symposium sizes, the target size is around 40-60 participants.

Lack of a long-term ongoing series of activities on the topic. The Spring Symposium Series is intended to nurture emerging communities and topics, so topics that already have yearly conferences or workshops are inappropriate.

An appropriate organizing committee. The organizing committee should have (1) good technical knowledge of the topic, (2) good organizational skills, and (3) connections to the various communities from which they intend to draw participants. Committees for cross-disciplinary symposia must adequately represent all the disciplines to be covered by the symposium.

Accepted proposals will be distributed as widely as possi- ble over the subfields of AI, and balanced between theoretical and applied topics. Symposia bridging theory and practice and those combining AI and related fields are particularly solicited.

Symposium proposals should be submitted as soon as possible, but no later than April 20,2012. Proposals that are submitted significantly before this deadline can be in draft form. Comments on how to improve and complete the proposal will be returned to the submitter in time for revisions to be made before the deadline. Notifications of acceptance or rejection will be sent to submitters around May 18, 2012. The submitters of accepted proposals will become the chair of the symposium, unless alternative arrangements are made. The symposium organizing committees will be responsible for:

8f Producing, in conjunction with the general chair, a Call for Participation and Registration Brochure for the symposium, which will be distributed to the AAAI membership

80 Additional publicity of the symposium, especially to potential audiences from outside the AAAI community

8 Reviewing requests to participate in the symposium and determining symposium participants

\& Preparing working notes for the symposium

\&o Scheduling the activities of the symposium

\&o Preparing a short review of the symposium, to be printed in AI Magazine

AAAI will provide logistical support, will take care of all local arrangements, and will arrange for reproducing and distributing the working notes. Please submit your symposium proposals by electronic mail (no postal submissions), and inquiries concerning symposia, to:

\&o Matthew E. Taylor Lafayette College

Easton, Pennsylvania, USA

taylorm@lafayette.edu

\section{March 25-27, 2013 Stanford University Stanford, California}

AAAI SSS-13 2275 East Bayshore Road, Suite 160 Palo Alto, CA 94303 650-328-3123 650-321-4457 (fax) sss13@aaai.org 